\title{
AMR simulations of the low $T /|W|$ bar-mode instability of neutron stars
}

\author{
Pablo Cerdá-Durán, Vicent Quilis, and José A. Font \\ Departamento de Astronomía y Astrofísica, Universidad de Valencia, Dr. Moliner \\ 50, 46100 Burjassot (Valencia), Spain
}

\begin{abstract}
It has been recently argued through numerical work that rotating stars with a high degree of differential rotation are dynamically unstable against bar-mode deformation, even for values of the ratio of rotational kinetic energy to gravitational potential energy as low as $\mathcal{O}(0.01)$. This may have implications for gravitational wave astronomy in high-frequency sources such as core collapse supernovae. In this paper we present high-resolution simulations, performed with an adaptive mesh refinement hydrodynamics code, of such low $T /|W|$ bar-mode instability. The complex morphological features involved in the nonlinear dynamics of the instability are revealed in our simulations, which show that the excitation of Kelvin-Helmholtz-like fluid modes outside the corotation radius of the star leads to the saturation of the bar-mode deformation. While the overall trends reported in an earlier investigation are confirmed by our work, we also find that numerical resolution plays an important role during the long-term, nonlinear behaviour of the instability, which has implications on the dynamics of rotating stars and on the attainable amplitudes of the associated gravitational wave signals.
\end{abstract}

Key words: gravitational waves, hydrodynamics, instabilities, stars: neutron stars: rotation

PACS: 97.60.Jd, 04.30.-w, 95.30.Lz

\section{Introduction}

Neutron stars following a core collapse supernova are rotating at birth and can be subject to various nonaxisymmetric instabilities (see e.g. [1] for a review). Among those, if the rotation rate is high enough so that the ratio of rotational kinetic energy $T$ to gravitational potential energy $W, \beta \equiv T /|W|$, exceeds the critical value $\beta_{\mathrm{d}} \sim 0.27$, inferred from studies with incompressible Maclaurin spheroids, the star is subject to a dynamical bar-mode $(l=m=2$ 
$f$-mode) instability driven by hydrodynamics and gravity. Its study is highly motivated nowadays as such an instability bears important implications in the prospects of detection of gravitational radiation from newly-born rapidly rotating neutron stars.

Simulations of the dynamical bar-mode instability are available in the literature, both using simplified models based on equilibrium stellar configurations perturbed with suitable eigenfunctions $[2,3,4,5]$, and more involved models for the core collapse scenario $[6,7,8,9]$, and in either case both in Newtonian gravity and general relativity. Due to its superior simplicity the former approach has received much more attention, notwithstanding that the conclusions drawn from perturbed stellar models may not be straightforwardly extended to the collapse scenario.

Newtonian simulations of triaxial instabilities following core collapse were first performed by [6]. These showed that the bar-mode instability sets in when $\beta \gg 0.27$ and when the progenitor rotates rapidly and highly differentially. Such conditions are met when the (artificial) depletion of internal energy to trigger the collapse is large enough to produce a very compact core for which a significant spun-up can be achieved. More recently, three-dimensional simulations of the core collapse of rotating polytropes in general relativity have been performed by [7]. These authors studied the evolution of the bar-mode instability starting with axisymmetric core collapse initial models which reached values of $\beta \sim 0.27$ during the infall phase. These simulations showed that the maximum value of $\beta$ achieved during collapse and bounce depends strongly on the velocity profile, the total mass of the initial core, and on the equation of state. In agreement with the findings from the Newtonian simulations of [6], the bar-mode instability sets in if the progenitor rotates rapidly $(0.01 \leq \beta \leq 0.02)$ and has a high degree of differential rotation. In addition, the artificial depletion of pressure and internal energy to trigger the collapse, leading to a compact core which subsequently spins up, also plays a key role in general relativity for a noticeable growth of the bar-mode instability.

Whether the requirements inferred from numerical simulations are at all met by the collapse progenitors remains unclear. As shown by [10] magnetic torques can spin down the core of the progenitor, which leads to slowly rotating neutron stars at birth $(\sim 10-15 \mathrm{~ms})$. The most recent, state-of-the-art computations of the evolution of massive stars, which include angular momentum redistribution by magnetic torques and spin estimates of neutron stars at birth $[11,12]$, lead to core collapse progenitors which do not seem to rotate fast enough to guarantee the unambiguous growth of the canonical bar-mode instability. Rapidly-rotating cores might be produced by an appropriate mixture of high progenitor mass $\left(M>25 M_{\odot}\right)$ and low metallicity $(\mathrm{N}$. Stergioulas, private communication). In such case the progenitor could by-pass the Red Supergiant phase in which the differential rotation of the core produces a 
magnetic field by dynamo action which couples the core to the outer layers of the star, transporting angular momentum outwards and spinning down the core. According to [13] about $1 \%$ of all stars with $M>10 M_{\odot}$ will produce rapidly-rotating cores.

On the other hand, Newtonian simulations of the bar-mode instability from perturbed equilibrium models of rotating stars have shown that $\beta_{\mathrm{d}} \sim 0.27$ independent of the stiffness of the equation of state provided the star is not strongly differentially rotating. The relativistic simulations of [5] yielded a value of $\beta \sim 0.24-0.25$ for the onset of the instability, while the dynamics of the process closely resembles that found in Newtonian theory, i.e. unstable models with large enough $\beta$ develop spiral arms following the formation of bars, ejecting mass and redistributing the angular momentum. As the degree of differential rotation becomes higher Newtonian simulations have also shown that $\beta_{\mathrm{d}}$ can be as low as 0.14 [14]. More recently $[15,16]$ have reported that rotating stars with an extreme degree of differential rotation are dynamically unstable against bar-mode deformation even for values of $\beta$ of $\mathcal{O}(0.01)$.

Given its recent discovery and its potential astrophysical implications for postbounce core collapse dynamics and gravitational wave astronomy, we present in this paper high resolution simulations of such low $T /|W|$ bar-mode instabilities. This work is further motivated in the light of the few numerical simulations available in the literature. Our main goal is to revisit the simulations by [15] on the low $T /|W|$ bar-mode instability, and particularly to check how sensitive the onset and development of the instability is to numerical issues such as grid resolution. To this aim we perform Newtonian hydrodynamical simulations of a subset of models analyzed by [15] using an adaptive mesh refinement (AMR) code [17] which allows us to perform such three dimensional simulations with the highest resolution ever used. Our simulations reveal the complex morphological features involved in the nonlinear dynamics of the instability, where the excitation of Kelvin-Helmholtz-like fluid modes influences the saturation of the bar-mode deformation. We advance that while the overall trends found by [15] are confirmed by our work, the resolution employed in the simulations does play a key role for the long-term behaviour of the instability and for the nonlinear dynamics of rotating stars, which has implications on the attainable amplitudes of the associated gravitational wave signals. We note that we plan to upgrade the existing AMR code to account for the effects of magnetic fields in order to attempt the current study in a more realistic setup. The present work is a step towards that goal.

The paper is organized as follows: Section 2 gives a brief overview of the equations to solve. Their solution is outlined in Section 3 which also contains the bare details of the AMR code. The results of the simulations are discussed in Section 4. Finally Section 5 presents our conclusions. 


\section{Mathematical framework}

The evolution of a self-gravitating ideal fluid in the Newtonian limit is described by the hydrodynamics equations and Poisson's equation:

$$
\begin{gathered}
\frac{\partial \rho}{\partial t}+\nabla \cdot(\rho \mathbf{v})=0 \\
\frac{\partial \mathbf{v}}{\partial t}+(\mathbf{v} \cdot \nabla) \mathbf{v}=-\frac{1}{\rho} \nabla p-\nabla \phi \\
\frac{\partial E}{\partial t}+\nabla \cdot[(E+p) \mathbf{v}]=-\rho \mathbf{v} \nabla \phi \\
\nabla^{2} \phi=4 \pi G \rho
\end{gathered}
$$

where $\mathbf{x}, \mathbf{v}=\frac{d \mathbf{x}}{d t}=\left(v_{x}, v_{y}, v_{z}\right)$, and $\phi(t, \mathbf{x})$ are, respectively, the Eulerian coordinates, the velocity, and the Newtonian gravitational potential. The total energy density, $E=\rho \epsilon+\frac{1}{2} \rho v^{2}$, is defined as the sum of the thermal energy, $\rho \epsilon$, where $\rho$ is the mass density and $\epsilon$ is the specific internal energy, and the kinetic energy (where $v^{2}=v_{x}^{2}+v_{y}^{2}+v_{z}^{2}$ ). Pressure gradients and gravitational forces are the responsible for the evolution. An equation of state $p=p(\rho, \epsilon)$ closes the system. We use an ideal gas equation of state $p=(\Gamma-1) \rho \epsilon$ with $\Gamma=2$.

The hydrodynamics equations, Eqs. (1-3), can be rewritten in flux-conservative form:

$$
\frac{\partial \mathbf{u}}{\partial t}+\frac{\partial \mathbf{f}(\mathbf{u})}{\partial x}+\frac{\partial \mathbf{g}(\mathbf{u})}{\partial y}+\frac{\partial \mathbf{h}(\mathbf{u})}{\partial z}=\mathbf{s}(\mathbf{u})
$$

where $\mathbf{u}$ is the vector of unknowns (conserved variables):

$$
\mathbf{u}=\left[\rho, \rho v_{x}, \rho v_{y}, \rho v_{z}, E\right]
$$

The three flux functions $\mathbf{F}^{\alpha} \equiv\{\mathbf{f}, \mathbf{g}, \mathbf{h}\}$ in the spatial directions $x, y, z$, respectively, are defined by

$\mathbf{f}(\mathbf{u})=\left[\rho v_{x}, \rho v_{x}^{2}+p, \rho v_{x} v_{y}, \rho v_{x} v_{z},(E+p) v_{x}\right]$

$\mathbf{g}(\mathbf{u})=\left[\rho v_{y}, \rho v_{x} v_{y}, \rho v_{y}^{2}+p, \rho v_{y} v_{z},(E+p) v_{y}\right]$

$\mathbf{h}(\mathbf{u})=\left[\rho v_{z}, \rho v_{x} v_{z}, \rho v_{y} v_{z}, \rho v_{z}^{2}+p,(E+p) v_{z}\right]$

and the source terms $\mathbf{s}$ are given by 
Table 1

Overview of the initial models and results of the simulations. The rows report the name of the model, the ratio of equatorial-to-polar radii $\left(r_{e} / r_{p}\right)$, the degree of differential rotation $(\hat{A})$, the ratio of kinetic to potential energy $(T /|W|)$, the size of the computational grid $(L)$ and the location of the corotation radius $\left(r_{c}\right)$ for the two resolutions used: high (AMR $\mathrm{H})$ and low (AMR L). In models $\mathrm{R} 1 \mathrm{H}$ and $\mathrm{R} 2 \mathrm{H}$ the corotation radius lies outside the star. The real (frequency) and imaginary (growth rate) parts of the bar-mode $\sigma_{2}$ are shown, for the low and high resolution simulation in comparison with the numerical results and linear analysis by [15]. Note that for model D3 no linear analysis results are available.

\begin{tabular}{lcccccc}
\hline \hline Model & & $\mathrm{D} 1$ & $\mathrm{D} 2$ & $\mathrm{D} 3$ & $\mathrm{R} 1$ & $\mathrm{R} 2$ \\
\hline$r_{e} / r_{p}$ & & 0.805 & 0.605 & 0.305 & 0.305 & 0.255 \\
$\hat{A}$ & & 0.3 & 0.3 & 0.3 & 1.0 & 1.0 \\
$T /|W|$ & & 0.039 & 0.085 & 0.149 & 0.253 & 0.275 \\
$L / r_{e}$ & & 4.06 & 3.73 & 3.21 & 4.25 & 4.03 \\
\hline$r_{c} / r_{e}$ & AMR L & 0.38 & 0.47 & 0.58 & & \\
& AMR H & 0.36 & 0.48 & 0.56 & - & - \\
\hline $\operatorname{Re}\left(\sigma_{2}\right) / \Omega_{0}$ & AMR L & 0.76 & 0.58 & 0.41 & & \\
& AMR H & 0.81 & 0.55 & 0.43 & - & 0.82 \\
& Shibata & 0.80 & 0.60 & 0.45 & 0.92 & 0.75 \\
& linear & 0.80 & 0.58 & - & 0.92 & 0.75 \\
\hline $\operatorname{Im}\left(\sigma_{2}\right) / \Omega_{0}$ & AMR L & 0.0042 & 0.0154 & 0.0200 & & \\
& AMR H & 0.0089 & 0.0190 & 0.0240 & 0.0005 & 0.1960 \\
& Shibata & $0.009-0.013$ & $0.019-0.021$ & 0.013 & $<0.002$ & 0.23 \\
& linear & 0.015 & 0.021 & - & $<0.002$ & 0.20 \\
\hline \hline
\end{tabular}

$\mathbf{s}(\mathbf{u})=\left[0,-\rho \frac{\partial \phi}{\partial x},-\rho \frac{\partial \phi}{\partial y},-\rho \frac{\partial \phi}{\partial z}, \rho v_{x} \frac{\partial \phi}{\partial x}-\rho v_{y} \frac{\partial \phi}{\partial y}-\rho v_{z} \frac{\partial \phi}{\partial z}\right]$

System (5) is a three-dimensional hyperbolic system of conservation laws with sources $\mathbf{s}(\mathbf{u})$. 


\section{Numerical approach}

For our study of the low $T /|W|$ bar-mode instability we perform high-resolution simulations of rotating neutron stars using a Newtonian AMR hydrodynamics code called MASCLET [17]. The implementation of the AMR technique in the code follows the procedure developed by [18]. The hydrodynamics equations are solved using a high-resolution shock-capturing scheme based upon Roe's Riemann solver and second-order cell reconstruction procedures, while Poisson's equation for the gravitational field is solved using multigrid techniques. The accuracy and performance of the MASCLET code has been assessed in a number of tests [17]. We note that the code was originally designed for cosmological applications, and here it is applied to simulations of self-gravitating stellar objects for the first time.

The simulations are performed with two different grid resolutions. The low resolution grid consists of a box of size $L$ with $128^{3}$ zones, yielding a fixed resolution of $L / 128$. We note that the effective resolution of our coarse grid is comparable to that used by [15]. Correspondingly, the high resolution grid consists of a base coarse grid of $128^{3}$ cells, and one level of refinement composed of patches with maximum size of $64^{3}$ cells ( $32^{3}$ coarse cells). This yields a grid resolution on the finest grid of $L / 256$. This resolution is enough to resolve the structures simulated, and hence no deeper refinement levels are needed. The patches are dynamically allocated covering those regions of the star where the highest resolution is required (highest densities). Typically only one patch is needed for spheroidal models, and 4-8 in models with toroidal topology. The use of AMR techniques in our high resolution simulations, allows us to save about a factor 4 in CPU time and memory with respect to a unigrid simulation with $256^{3}$ cells. No symmetries are imposed in the simulations. To the best of our knowledge, in the investigations of the bar-mode instability performed by previous groups, grid resolutions as high as the ones we use here were never employed.

As customary in grid-based codes $[19,20]$ the vacuum surrounding the star is filled with a tenuous numerical atmosphere with density $\rho / \rho_{\max } \approx 10^{-12}$ and zero velocities, $\rho_{\max }$ being the maximun density. Every grid cell with $\rho / \rho_{\max }<10^{-6}$ is reset to the atmosphere values. A correct treatment of the atmosphere is essential for an accurate description of the stellar dynamics and correct computation of the growth rates of unstable modes. We have checked that values for the atmosphere higher than those we chose or a free evolution of the atmosphere altogether, lead to remarkable changes in the mode behaviour, growth rates, and frequencies. We have also checked that lower values for the atmosphere do not produce those changes, which ensures that our evolutions are not affected by the atmosphere values used in the simulations. 


\section{Results}

\subsection{Initial data}

Differentially rotating stellar models in equilibrium are built according to the method of [21], and used as initial data for the AMR evolution code. The stars obey a polytropic equation of state $P=K \rho^{\Gamma}$ with index $\Gamma=2$. As [15] the profile of the angular velocity $\Omega$ is given by

$\Omega=\frac{\Omega_{0} \hat{A}^{2}}{\left(\varpi / r_{e}\right)^{2}+\hat{A}^{2}}$,

where $r_{e}$ is the equatorial radius of the star, $\Omega_{0}$ is the central angular velocity, $\varpi$ is the distance to the rotation axis, and $\hat{A}$ parametrizes the degree of differential rotation, from $\hat{A} \ll 1$ for highly differentially rotating stars to $\hat{A} \rightarrow \infty$ for rigidly rotating stars. For comparison purposes these parameters are chosen as in some of the models of [15], and are summarized in Table 1. Models labelled $\mathrm{D}$ rotate with a high degree of differential rotation, as $\hat{A}=0.3$, and may therefore be subject to the low $T /|W|$ bar-mode instability. We also consider models almost rigidly rotating, labelled $\mathrm{R}$, prone to experience the "classical" bar-mode instability. Labels $\mathrm{L}$ and $\mathrm{H}$ in the models refer to low and high resolution respectively.

Following [15] we perturb the initial density profile $\rho^{(0)}$ according to

$\rho=\rho^{(0)}\left(1+\delta \frac{x^{2}-y^{2}}{r_{e}^{2}}\right)$

the perturbation of the pressure given by the equation of state accordingly. A perturbation amplitude $\delta=0.1$ is used in all our simulations. As we show below this form of the perturbation excites the $l=m=2$ bar-mode. In addition, grid discretization can leak small amounts of energy to all other possible modes, which could in principle grow provided they were unstable and the simulations were carried on for sufficiently long times.

\subsection{Stability analysis}

To compare with [15] we calculate the distortion parameters $\eta_{+}$and $\eta_{\times}$(and $\left.\eta=\left(\eta_{+}^{2}+\eta_{\times}^{2}\right)^{1 / 2}\right)$ defined as 


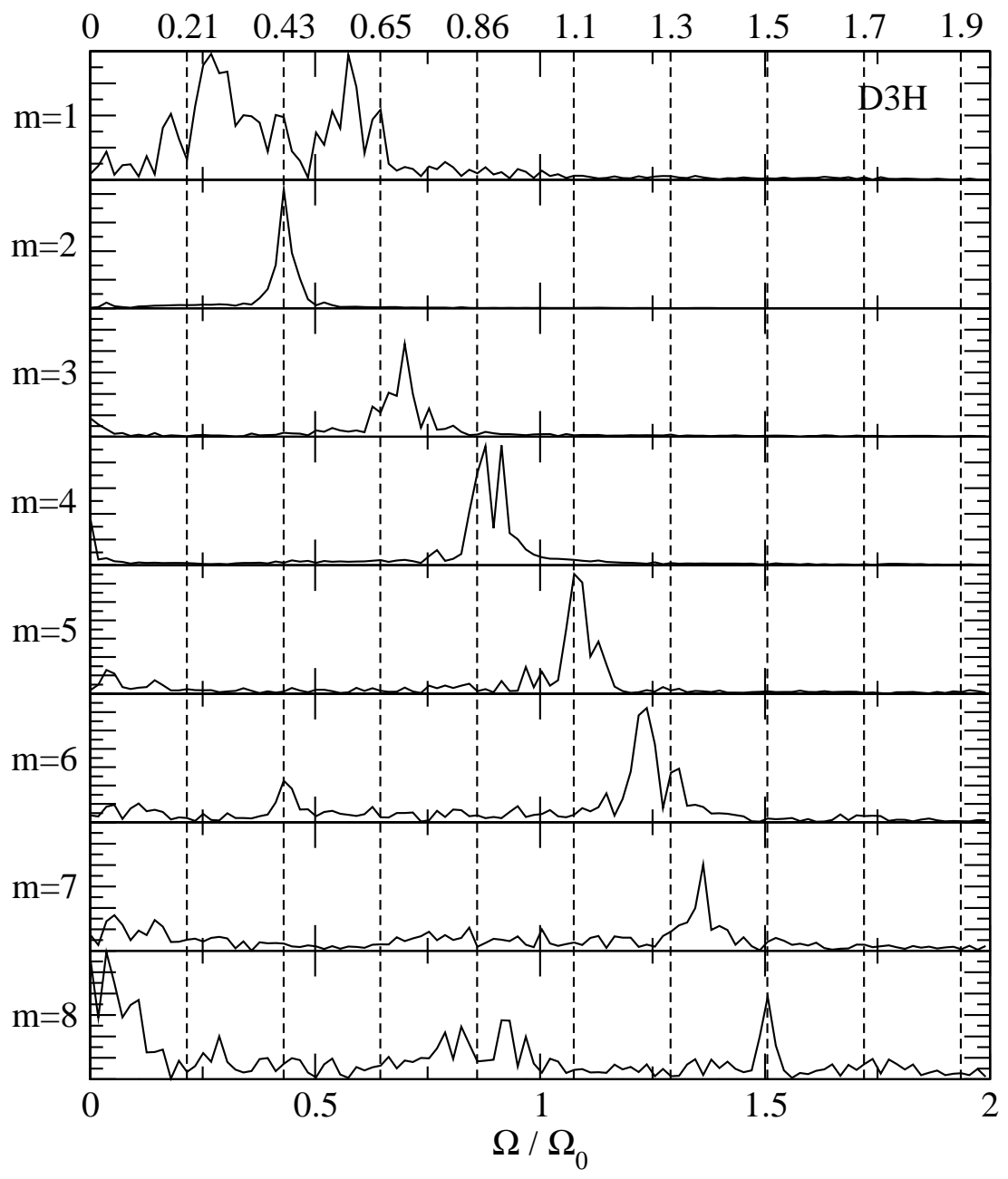

Fig. 1. Power spectra of $\mathcal{A}_{m}$ from $m=1$ to $m=8$ for model D3H.

$\eta_{+} \equiv \frac{I_{x x}-I_{y y}}{I_{x x}+I_{y y}}, \quad \eta_{\times} \equiv \frac{2 I_{x y}}{I_{x x}+I_{y y}}$,

where $I_{i j}(i, j=x, y, z)$ is the mass-quadrupole moment

$I_{i j}=\int d \mathbf{x}^{3} \rho x^{i} x^{j}$

For the study of the growth rate and interaction of the different angular modes within the star is useful to calculate the global quantity

$A_{m}=\int d \mathbf{x}^{3} \rho(\mathbf{x}) e^{-i m \varphi}$

and $\mathcal{A}_{m} \equiv A_{m} / A_{0}$. We follow the time evolution of modes with $m$ ranging from 1 to 8 . Since our initial equilibrium models are axisymmetric and have equatorial plane symmetry, all $\mathcal{A}_{m}$ are zero initially, but once perturbed all 


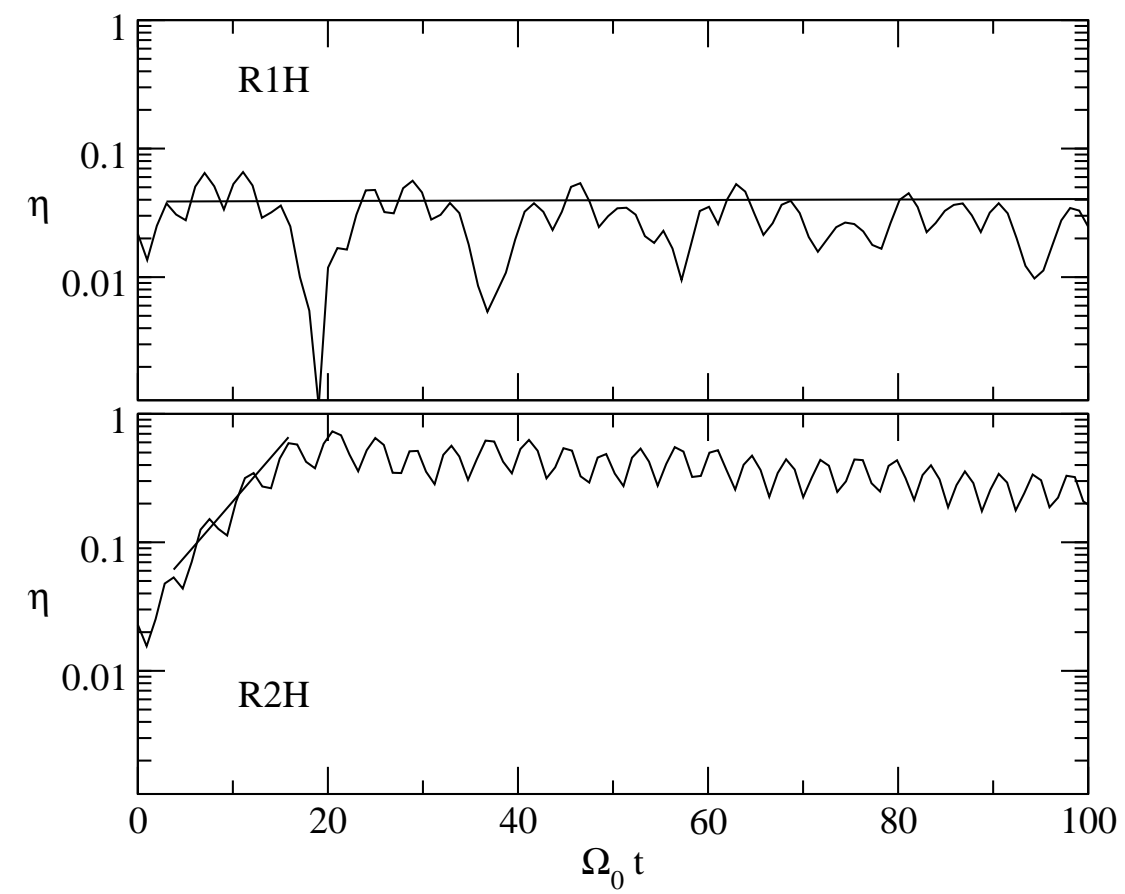

Fig. 2. Evolution of $\eta$ for models R1H (upper panel) and R2H (lower panel). Exponential fits to the peaks in the growing phase are overplotted as solid lines.

initial models exhibit a dominant $m=2$ component. Assuming that the modes behave as $e^{-i\left(\sigma_{m} t-m \varphi\right)}$, the real part of $\sigma_{m}$ can be obtained by Fourier transforming $\mathcal{A}_{m}$. In particular $\operatorname{Re}\left(\sigma_{2}\right)$, the bar-mode frequency, can be extracted from either $\mathcal{A}_{2}$ or $\eta$ as both represent the same mode. This is the dominant mode in all our simulations and its frequency and growth rate are given in Table 1. The latter corresponds to the imaginary part of $\sigma_{2}$, which is calculated fitting an exponential to the peak values of $\eta$ in the growing phase of the evolution until the modes saturate. Other modes are also identified in the simulations for values of $\mathcal{A}_{m}$ with lower amplitudes. We have checked that these modes are harmonics of the $l=m=2$ mode so that they follow to good accuracy the relation $\sigma_{m}=m \sigma_{p}, \sigma_{p}$ being the pattern frequency, calculated as $\sigma_{p}=\sigma_{2} / 2$. This is shown for model D3H in Fig. 1 which displays the spectrum of $\mathcal{A}_{m}$ from $m=1$ to $m=8$ (in arbitrary units). The vertical dashed lines in this figure indicate the location of the integer multiples of the pattern frequency $\sigma_{p}$, their values indicated on the axis at the top of the figure. Each spectrum for each mode is normalized to its own maximum for plotting purposes. Note that the lower the mode amplitude the noisier the spectrum and the less accurate the relation $\sigma_{m}=m \sigma_{p}$.

For the models of our sample subject to the "clasical" bar-mode deformation ( $\mathrm{R} 1 \mathrm{H}$ and $\mathrm{R} 2 \mathrm{H}$ ), our simulations yield a value of $\beta$ between 0.253 and 0.275 , in good agreement with the critical value for the onset of the dynamical barmode instability. Model R1H is stable and model $\mathrm{R} 2 \mathrm{H}$ is unstable. The growth rates and frequencies reported in Table 1 agree with those of [15]. Note that 


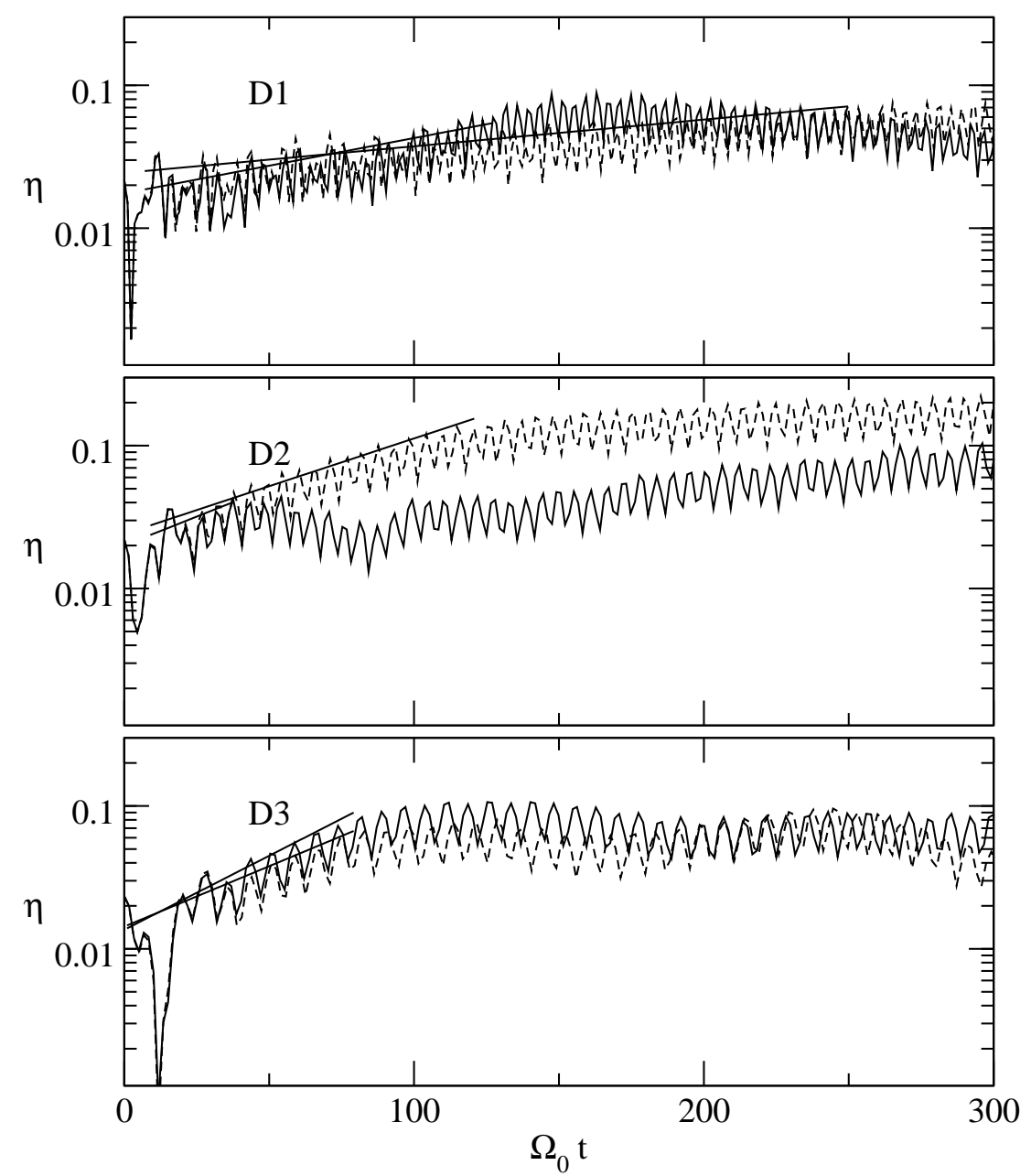

Fig. 3. Evolution of $\eta$ for models D1 (upper panel), D2 (central panel) and D3 (lower panel). Dashed lines correspond to low resolution and solid lines to high resolution. Exponential fits to the peaks in the growing phase are overplotted as solid lines.

for model R1H, which is stable, the frequency for the $m=2$ mode cannot be computed. The time evolution of $\eta$ for these two models is displayed in Fig. 2. For the unstable model R2H, our simulations show the formation of a bar which saturates for values of $\eta_{+}$and $\eta_{\times}$close to 1 , i. e. in the full nonlinear regime.

Fig. 3 shows the time evolution of $\eta$ for models D in our sample, prone to suffer the low $T /|W|$ bar-mode instability. Solid lines correspond to high resolution simulations and dashed lines to low resolution. For all three models the pattern frequencies $\sigma_{p}$ are such that there exists a corotation radius inside the star, i.e. a radius at which the bar-mode rotates with the same angular velocity as the fluid. The location of the corotation radius for all models of our sample is reported in Table 1. As recently discussed by [22] the existence of such corotation radius is a potential requirement for the ocurrence of the instability. As becomes clear from Fig. 3, all models are unstable but grid resolution has 
an important effect on the saturation of the instability once the nonlinear phase has been reached, as well as in the long-term dynamics of the stars.

In the linear phase of models $\mathrm{D} 1 \mathrm{H}$ and $\mathrm{D} 2 \mathrm{H}$, the growth rates and frequencies agree with the results of [15] in both, the numerical simulations and the linear analysis (see Table 1). In the linear phase of model D3H, our frequencies are similar to the numerical results of [15], although our growth rates are about a factor two larger. We emphasize that no results are reported in the linear analysis for this model in the work of [15], and therefore this discrepancy can be an effect of the resolution used or of the characteristics of each numerical code. Increasing resolution leads to similar results in the frequencies but to higher growth rates.

In the nonlinear phase, models D1 and D3 behave similarly for the two resolutions used (see Fig. 3), and also similarly to the results by [15] (compare with Fig. 3 of that paper). For model D2 we observe a radical change of behavior in the nonlinear phase of the mode evolution depending on the grid resolution. This has implications on the long-term dynamics of the star and, in particular, on the attainable amplitudes of the gravitational radiation emitted, as we discuss below.

It is worth mentioning the possibility that the unstable mode at the start of model D2H might excite some other mode in the corotation band, which could not otherwise be excited for lower grid resolution. As discussed by $[23,24]$ in their study of differentially rotating shells, there are many zero-step modes in the band, so that the whole continuous spectrum could potentially be excited. In such case these modes would have very slow power-law growth.

For all our models we have checked mass conservation along the evolution. The worst results are obtained for model D3H, for which mass is conserved within $2.5 \%$ error when the instability saturates. At the end of the simulation (after 48 orbital periods and 25000 iterations in the coarsest grid) the error has grown to only $6 \%$. For all other models mass conservation is even more accurate. Note that these errors are within the round-off error of the code, and it is not related to the conservation properties of the numerical scheme itself. For a regular grid with $128^{3}$ cells and a simulation employing 25000 iterations, the accumulated round-off error (binomial distribution) using single-precision arithmetics, is about $\sqrt{128^{3} \times 25000} \times 10^{-8}=0.0023=0.23 \%$. Correspondingly, for a $256^{3}$ grid (with twice the number of iterations for the simulation) the error is about $0.9 \%$. Taking into account that this error affects the nonlinear evolution of the system, it is not surprising to have an error at the level of a few percent by the end of our high resolution simulations, for all conserved quantities.

Figure 4 shows the evolution of $\mathcal{A}_{m}$ for model D3 and for $m$ ranging from 1 to 8 for our two resolutions. According to this figure, the only two modes 


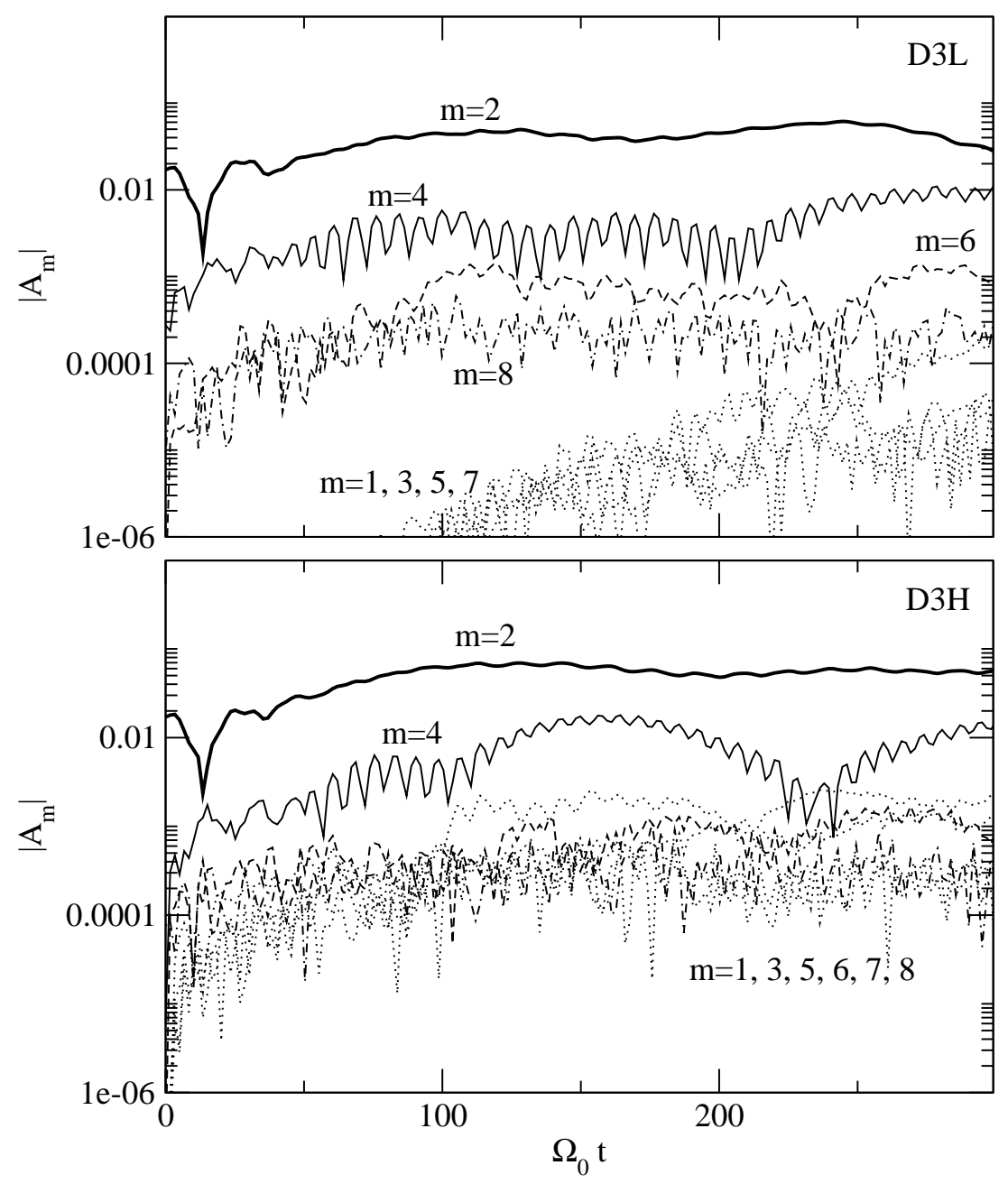

Fig. 4. Evolution of $\left|\mathcal{A}_{m}\right|$ for model D3 with low resolution (top) and high resolution (bottom). The $m=2$ mode is represented with thick solid line, $m=4$ with thin solid line, $m=6$ with dashed line, $m=8$ with dot-dashed line, and all other odd $m$ with dotted lines.

relevant for the dynamics of the star are $m=2$ and $m=4$. All other modes have smaller amplitudes and play no role in the dynamics. Note that for odd $m$ modes, the value of the integrated quantity $\mathcal{A}_{m}$, if close to zero, is extremely sensitive to very small numerical asymmetries, which are induced by the patch creation scheme of our AMR code. This explains the resolution differences in the initial values for odd $m$ modes in Fig. 4 (at $t=0$ they start off at $10^{-8}$ level for the low resolution simulation), although they saturate at the same value irrespective of the resolution.

An important diagnosis for the accuracy of the results is the location of the center of mass during an evolution. The round-off error of the numerical code imposes controlled errors in mass and linear momentum, which results in tiny displacements of the center of mass. However small (one numerical cell in our runs) this unphysical displacement may hinder the correct analysis of the 


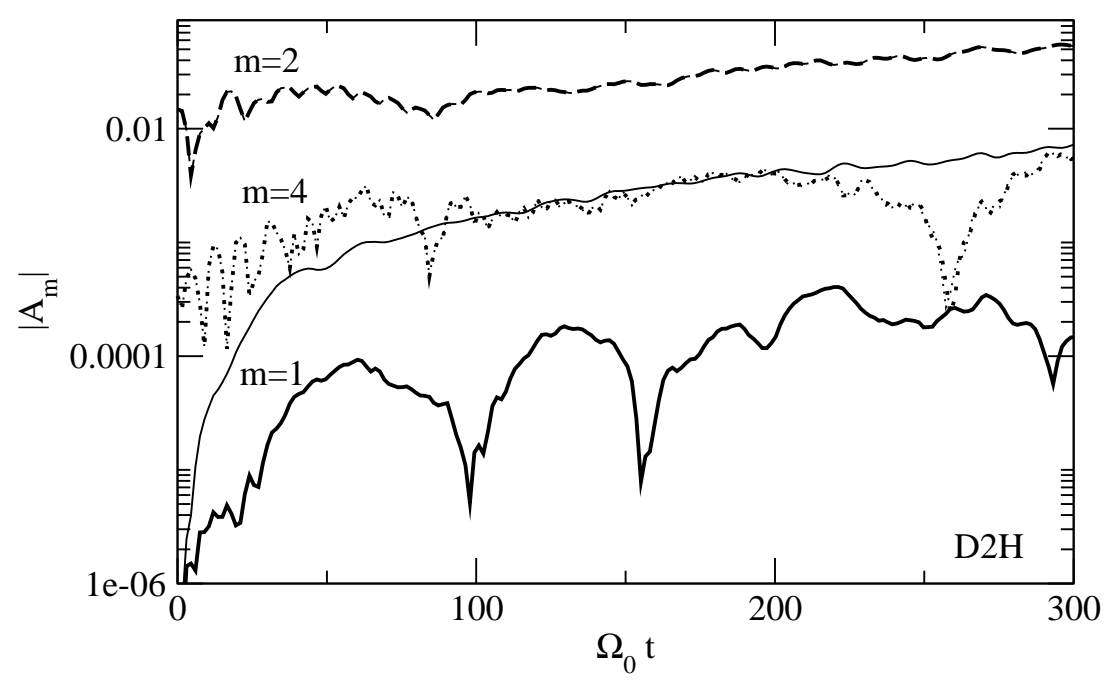

Fig. 5. Effects of the artificial displacement of the center of mass (of only one numerical cell) on the time evolution of $\left|\mathcal{A}_{1}\right|$ for model D2H. The thin solid line shows a fictitiuos evolution resulting from the numerical artifact originated by the center of mass displacement.

mode growth rates. For this reason all integrated quantities shown in Fig. 4 are computed after correcting for the displacement of the center of mass, $\mathbf{x}_{\text {new }}=\mathbf{x}_{\text {old }}-\mathbf{x}_{\mathrm{CM}}$, in a post-processing stage of the data analysis. Were this not done, a one-armed $m=1$ mode would grow much faster than it should to bring up fictitious features in the plots. This is shown for model D2H in Fig. 5. The thick solid line in this figure corresponds to the evolution of the $m=1$ mode taking into account the correction for the center of mass displacement, while the thin solid line is the corresponding evolution of this mode without the correction.

\subsection{Gravitational waves}

The growth and saturation of the instability is also imprinted on the gravitational waves emitted. The gravitational waveforms $h_{+}$and $h_{\times}$for models D1, D2, and D3, computed using the standard quadrupole formula, are shown in Fig. 6. For a source of mass $M$ located at a distance $R$ those waveforms can be calculated from the dimensionless waveform amplitudes $a_{+}$and $a_{\times}$as

$h_{+, \times}=a_{+, \times} \frac{\sin ^{2} \theta}{R} \frac{M^{2}}{r_{e}}$,

using $G=c=1$ units. The resulting chirp-like signal in all the models, particularly apparent for model D2L, indicates the presence of a bipolar distribution of mass within the star (see Sec. 4.4). 


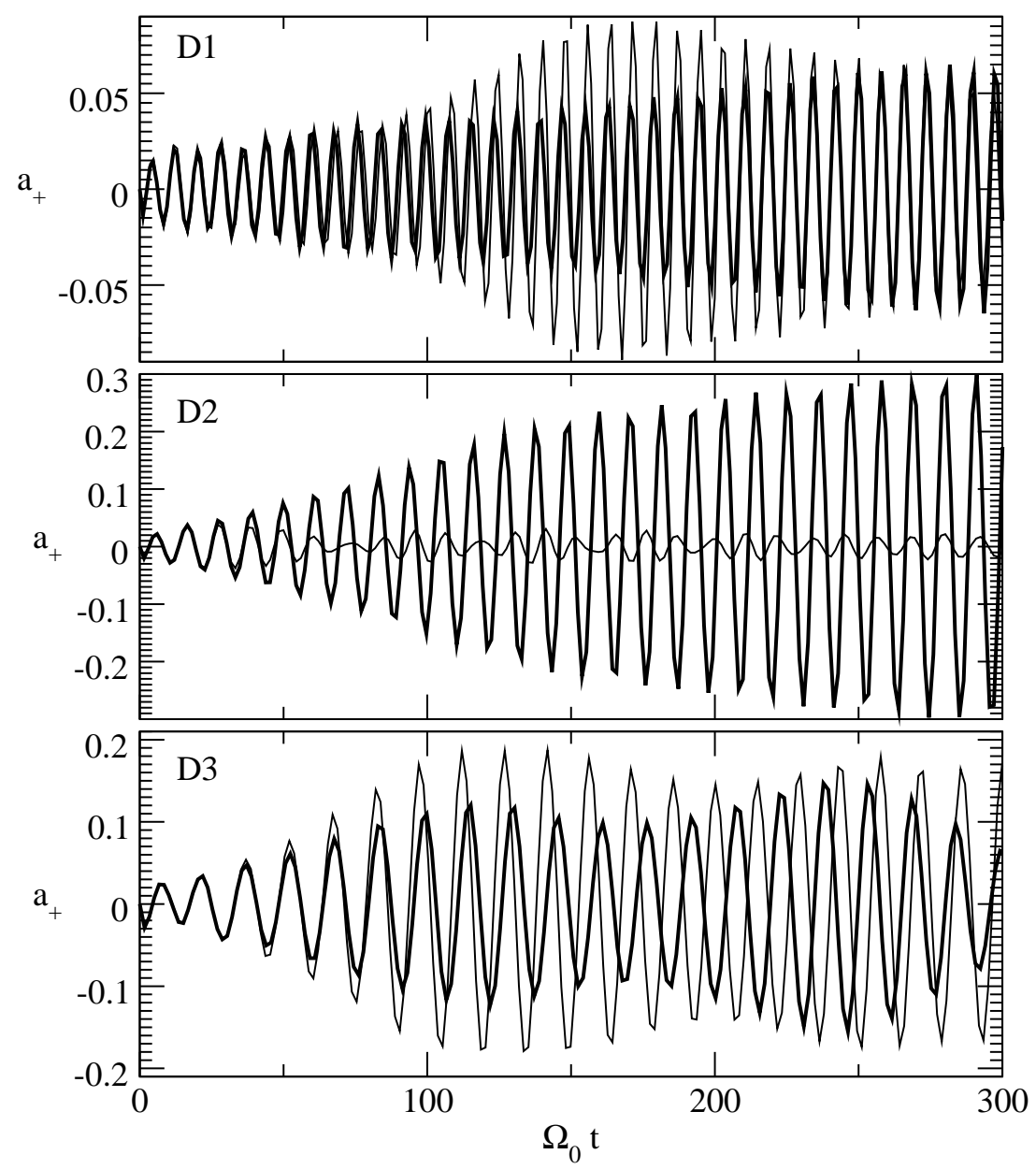

Fig. 6. Gravitational waves for models D1 to D3 extracted using the standard quadrupole formula. Thick (thin) solid lines correspond to low (high) resolution. Only the dimensionless waveform amplitude $a_{+}$is plotted.

As mentioned before, the effects of grid resolution on the evolution of the nonlinear phase of the bar-mode are imprinted on the gravitational waveforms. Thick solid lines in Fig. 6 are the waveforms which correspond to the lowresolution models, and thin solid lines to the high-resolution counterparts. The evolution of $\eta$ for model D3, displayed in Fig. 3, shows little deviations with grid resolution, and this translates into very similar gravitational wave patterns (bottom panel of Fig. 6), the differences becoming more noticeable in the nonlinear phase following saturation $\left(\Omega_{0} t \geq 75\right)$. For model D1 (top panel), the differences also become more apparent at later times during the evolution, in good agreement with the dissimilar behaviour of the matter dynamics in this model, as encoded in the evolution of $\eta$ in Fig. 3. As happens for model D3 the first few cycles of the gravitational waveform, when the mode is still in the linear phase, are accurately captured for both resolutions.

The major dependence of the waveform on the grid resolution is found for model D2. Again, the linear phase for the growth of the bar deformation is 


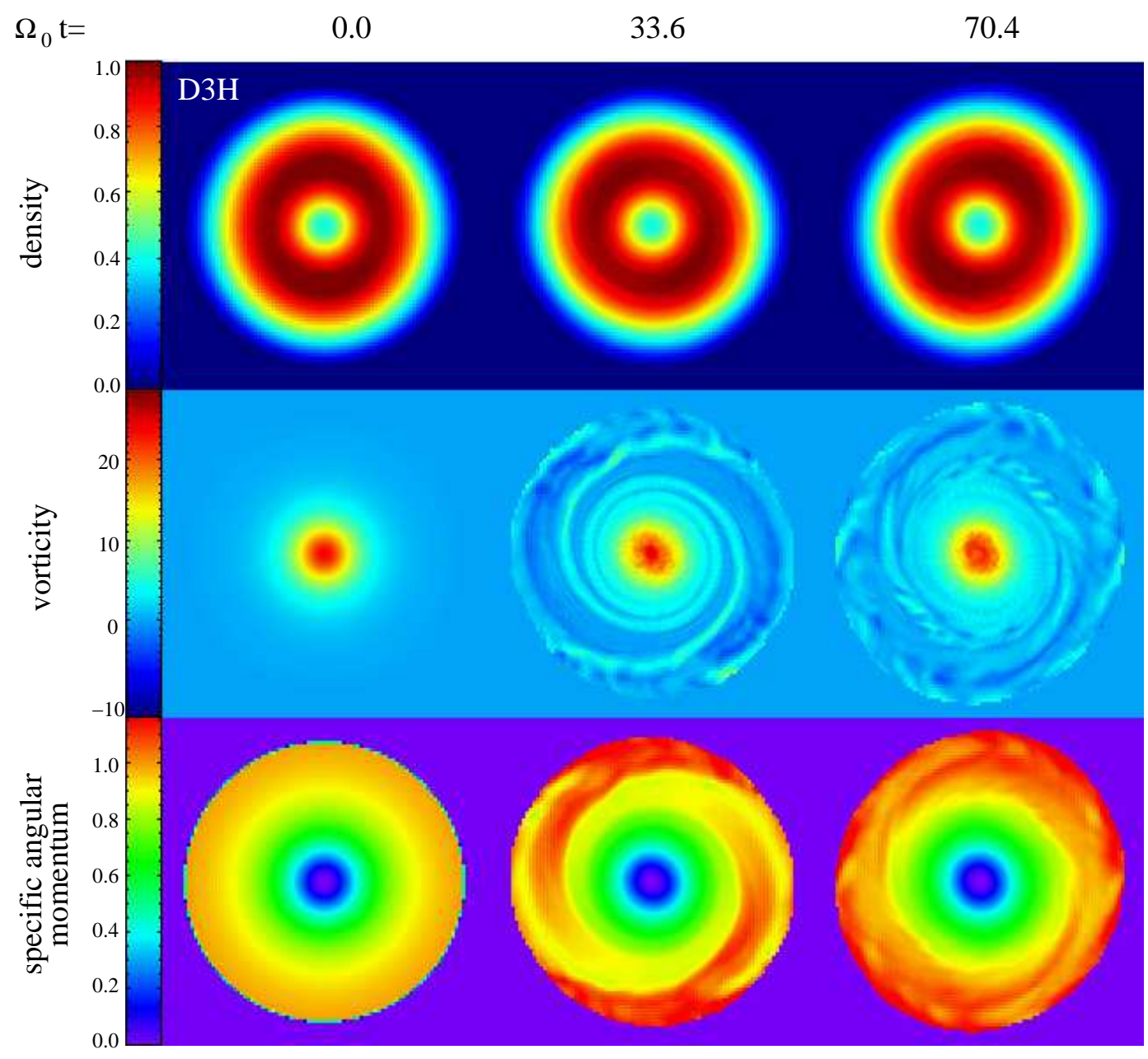

Fig. 7. Snapshots of the density, vorticity, and specific angular momentum, for model $\mathrm{D} 3 \mathrm{H}$, at three representative instants of the evolution. All snapshots show slices of the stars in the equatorial plane. Quantities are normalized as follows: $\rho / \rho_{\max }^{(0)}, r_{e} w^{\varphi} / v_{s}^{(0)}$, and $l^{\varphi} /\left(r_{e} v_{s}^{(0)}\right)$, where $v_{s}^{(0)}$ is the initial velocity at the surface of the star.

accurately captured irrespective of the resolution (and agrees with the perturbative results of [15]). This is signalled in the perfect overlapping of both gravitational waveforms during the first three cycles (see the middle panel of Fig. 6). However, the different nonlinear dynamics of the bar-mode deformation for this model, shown in the middle panel of Fig. 3, is severely imprinted on the gravitational waveform. Model D2H emits gravitational waves which have roughly one order of magnitude smaller amplitude than those computed for the corresponding low resolution model. 


\subsection{Morphology}

We next describe the morphological features encountered during the evolution of some representative models. Fig. 7 shows three snaphsots of the evolution of model D3H for the density (top), the azimuthal component of the vorticity,

$\vec{w}^{\varphi}=(\nabla \times \vec{v})^{\varphi}$ (middle), and the specific angular momentum, $\vec{l}=\vec{r} \times \vec{v}$ (bottom). From left to right the snapshots correspond to the initial time $\left(\Omega_{0} t=\right.$ $0)$, a time when the bar-mode instability is growing $\left(\Omega_{0} t=33.6\right)$, and the time when the instability saturates $\left(\Omega_{0} t=70.4\right)$. Only the equatorial plane of the stars is shown in all these plots. Animations of all simulations performed are available at www.uv.es/ cerdupa/bars/. We note that our AMR code is able to dynamically place patches (e. g. between 4 and 8 in the D3H model) and evolves the system with continuous matching between patches, as exemplified in Fig. 7.

The evolution of model D3H shows that as the $m=2$ mode grows the star develops an ellipsoidal shape which remains spinning beyond saturation. Since the low $\beta m=2$ mode saturates at lower values $(\eta \sim 0.1)$ than the classical bar-mode instability $(\eta \sim 1)$, no clear bars are visible in the density plot. At late times $\left(\Omega_{0} t>100\right)$ a "boxy" structure becomes apparent as the $m=4$ mode has grown to almost similar amplitude as the $m=2$ mode (see animations and Fig. 4). No other global features can be seen, consistent with the fact that $\left|\mathcal{A}_{m}\right| \ll 1$ for all modes other than $m=2$ and 4 . The vorticity plot shows that the $m=2$ mode at $\Omega_{0} t=33.6$ adopts the form of a two-armed spiral winding up around the central parts of the star. As the mode begins to saturate $\left(\Omega_{0} t=70.4\right)$ the spirals break apart into the outer layers in a turbulent flow reminiscent of the (shear) Kelvin-Helmholtz instability, and shock as they reach the atmosphere. These trends are also visible in the specific angular momentum plot.

The presence of a corotation radius, at $r / r_{e}=0.56$ for model $\mathrm{D} 3 \mathrm{H}$, seems to play a role in the growth and saturation of the instability, in agreement with the recent findings of [25]. As the bar-mode grows, pressure waves carry angular momentum outside the corotation radius, which is deposited in the outer layers of the star. This excites Kelvin-Helmholtz-like instabilities in the fluid that break the mode outside the corotation radius. When this happens the $m=2$ instability stops growing and no more angular momentum is extracted. Figure 8 shows late-time snapshots of the equatorial plane distribution of the density perturbation, i.e. $\left(\rho-\rho^{(0)}\right) / \rho_{\max }^{(0)}$, for models D2H and D3H. The times are chosen well inside the nonlinear and saturation phase of the instability. This figure helps to interpret the mode dynamics and its saturation along the lines mentioned before: During the evolution the density perturbations are shed in waves from the center towards the outer layers of the star. At late times, when the instability saturates, such shedding stops, and the density 


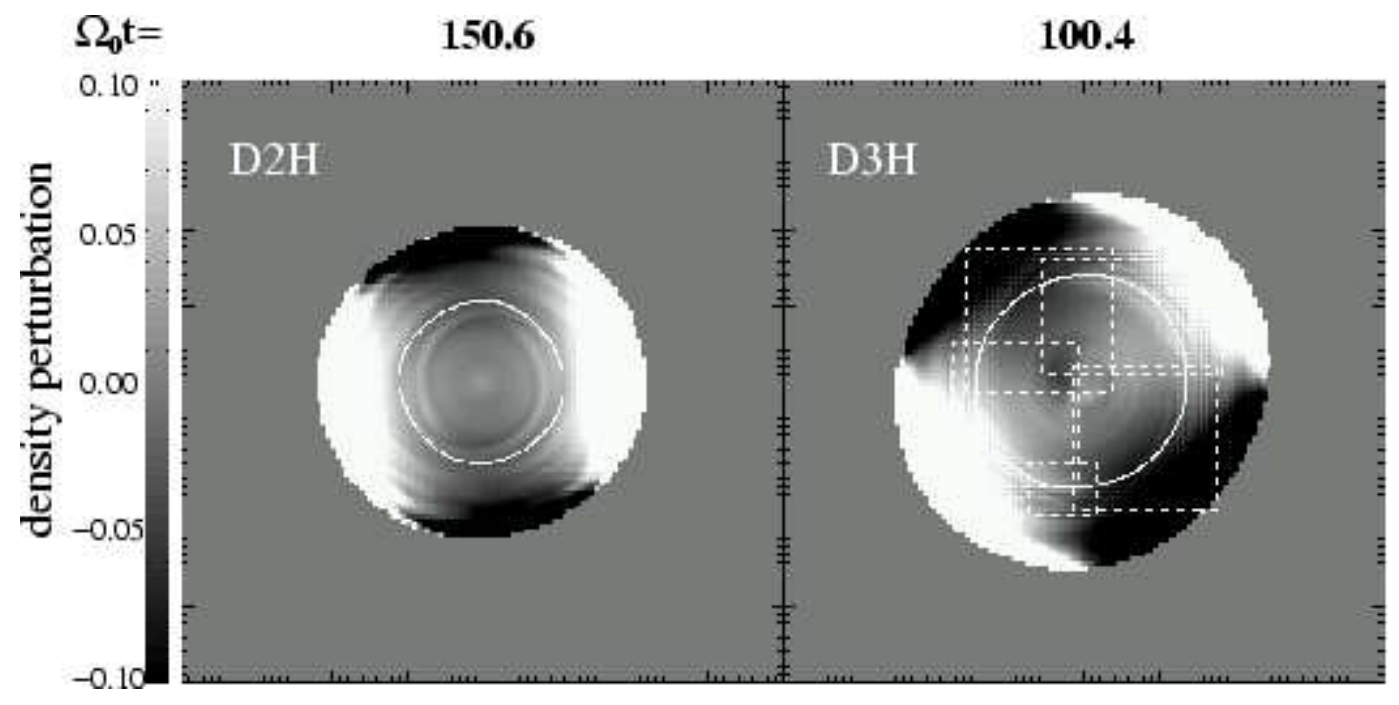

Fig. 8. Snapshots of the density perturbation at the equatorial plane for models D2H and $\mathrm{D} 3 \mathrm{H}$. The white solid curves indicate the location of the corotation radius. The white dashed boxes indicate the location of the patches for model $\mathrm{D} 3 \mathrm{H}$.

perturbation reaches the largest values outside the corotation radius (depicted with white solid lines in Fig. 8), for either model.

We note in passing that the corotation radius in all our high resolution models lies well inside the outer boundary of the finest box set up by the AMR refinement pattern. (see, e.g. the white dashed boxes depicted in the right panel of Fig. 8 indicating the location of the AMR patches for model D3H) This rules out the possibility of a numerical artifact resulting from the patch creation scheme of our AMR code being the cause for the different long-term evolution between low and high resolution models, particularly noticeable for model D2 in Fig. 3.

Finally, Fig. 9 shows a comparison between models D2L and D2H at $\Omega_{0} t=101$ (i.e. well within the nonlinear phase), to highlight the effects of the numerical resolution on the morphology. From top to bottom this panel shows a schlieren plot $(|\nabla \log \rho|), \vec{w}^{\varphi}$, and $\vec{l}$. The resolution differences in the evolution of model D2 become apparent from this figure. In particular, the "boxy" structure becomes much more clearly visible in the low resolution simulation (D2L), indicating an excessive growth rate of the $m=4$ mode. The presence of pressure waves is emphasized in the schlieren plot, very accurately captured in model D2H. Those waves, once the flow is driven to turbulence past the corotation radius, redistribute the angular momentum in the outer layers of model D2L in a much more pronounced way than for model D2H. 


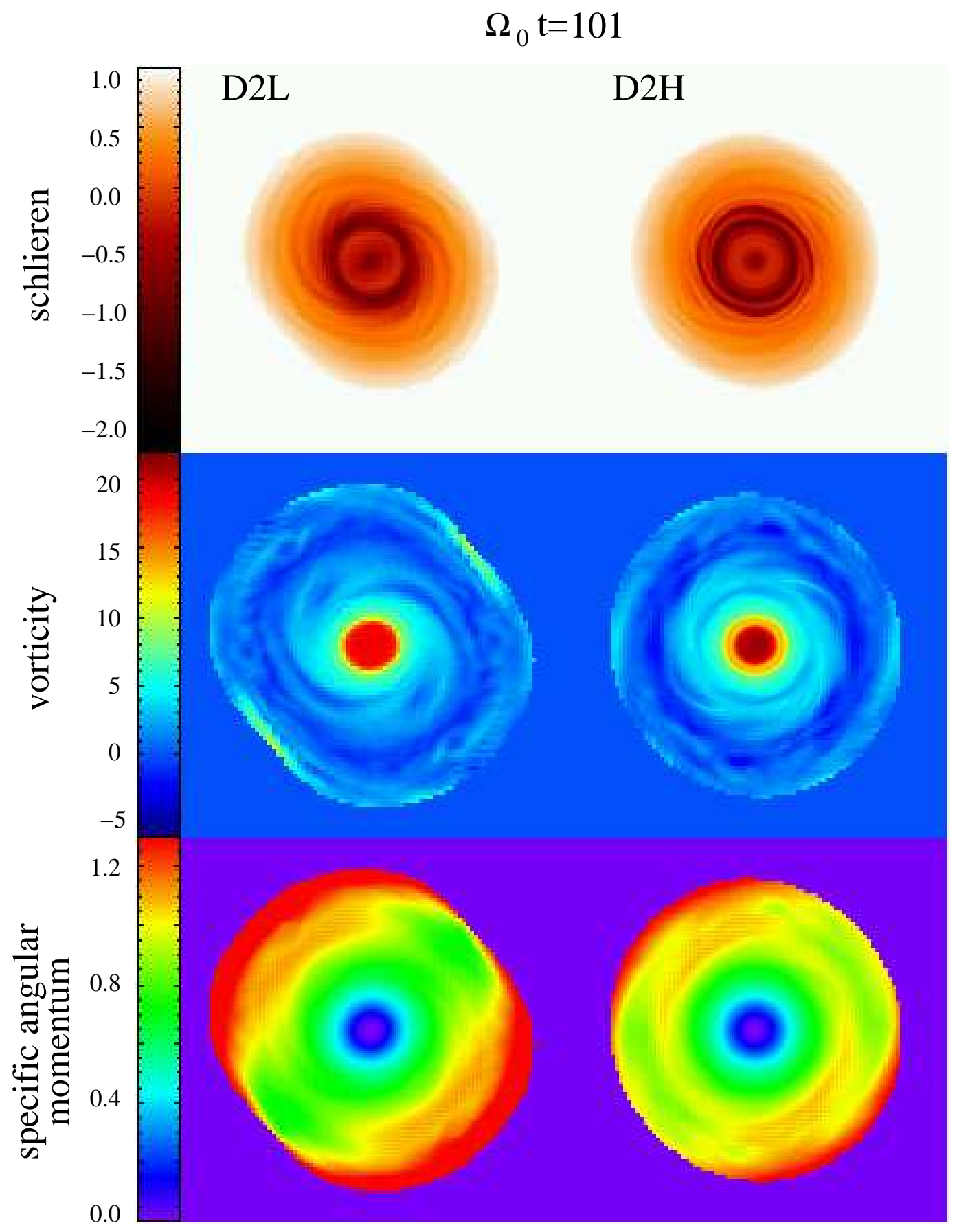

Fig. 9. Resolution comparison between models D2L and D2H once the instability has saturated. Only slices of the stars in the equatorial plane are shown.

\section{$5 \quad$ Summary and outlook}

We have presented AMR high-resolution simulations of the low $T /|W|$ barmode instability of extremely differentially rotating neutron stars. Our main motivation has been to revisit the simulations by [15] on such instability, 
assessing how sensitive the onset and development of the instability is to numerical issues such as grid resolution. We have addressed the importance of a correct treatment of delicate numerical aspects which may spoil threedimensional simulations in (Cartesian) grid-based codes, always hampered by insufficient resolution, namely the handling of the low-density atmosphere surrounding the star, the correction for the center of mass displacement, and the mass and momentum conservation properties of the numerical scheme. Our simulations have revealed the complex morphological features involved in the nonlinear dynamics of the instability. We have found that in the nonlinear phase of the evolution, the excitation of Kelvin-Helmholtz-like fluid modes outside the corotation radii of the stellar models leads to the saturation of the bar-mode deformation. While the overall trends reported in the investigation of [15] are confirmed by our work, the resolution used to perform the simulations may play a key role on the long-term behaviour of the instability and on the nonlinear dynamics of rotating stars, which has only become apparent for some specific models of our sample (namely model D2). This, in turn, has implications on the attainable amplitudes of the associated gravitational wave signals.

The work reported in this paper is a first step in our ongoing efforts of studying the dynamical bar-mode instability within the magnetized core collapse scenario.

\section{Acknowledgements}

The authors thank Harry Dimmelmeier, Nick Stergioulas, and Anna Wats for useful comments. Research supported by the Spanish Ministerio de Educación y Ciencia (MEC; grants AYA2004-08067-C03-01, AYA2003-08739-C0202, AYA2006-02570). VQ is a Ramón y Cajal Fellow of the Spanish MEC.

Computations performed at the Servei d'Informática de la Universitat de València (CERCA-CESAR).

\section{References}

[1] N. Stergioulas, Liv. Rev. Relativ. 6 (2003) 3

[2] J. E. Tohline, R. H. Durisen, \& M. McCollough, ApJ 298 (1985) 220

[3] J. L. Houser, J. M. Centrella, \& S. Smith, Phys. Rev. Lett. 72 (1994) 1314

[4] K. C. B. New, J. M. Centrella, \& J. E. Tohline, Phys. Rev. D 62 (2000) 064019

[5] M. Shibata, T. W. Baumgarte, \& S. L. Shapiro, ApJ 542 (2000) 453 
[6] M. Rampp, E. Müller, \& M. Ruffert, A\&A 332 (1998) 969

[7] M. Shibata, \& Y. Sekiguchi, Phys. Rev. D 71 (2005) 024014

[8] M. Saijo, Phys. Rev. D 71 (2005) 104038

[9] C. D. Ott, S. Ou, J. E. Tohline, \& A. Burrows, ApJ 625 (2005) L119

[10] H. C. Spruit \& E. S. Phinney, Nature 393 (1998) 139

[11] A. Heger, S. E. Woosley, \& H. C. Spruit, ApJ 626 (2005) 350

[12] C. D. Ott, A. Burrows, T. A. Thompson, E. Livne, \& R. Walder, ApJS 164 (2006) 130

[13] S. E. Woosley, \& A. Heger, ApJ 637 (2006) 914

[14] J. M. Centrella, K. C. B. New, L. L. Lowe, \& J. D. Brown, ApJ 550 (2001) L193

[15] M. Shibata, S. Karino, \& Y. Eriguchi, MNRAS 334 (2002) L27

[16] M. Shibata, S. Karino, \& Y. Eriguchi, MNRAS 343 (2003) 619

[17] V. Quilis, MNRAS 352 (2004) 1426

[18] M. J. Berger, P. Colella, J. Comp. Phys. 82 (1989) 64

[19] J. A. Font, M. Miller, W.-M. Suen, \& M. Tobias, Phys. Rev. D 61 (2000) 0044011

[20] M. D. Duez, P. Marronetti, S. L. Shapiro, \& T. W. Baumgarte, Phys. Rev. D 67 (2003) 024004

[21] Y. Eriguchi, \& E. Müller, A\&A, 147 (1984) 161

[22] A. L. Watts, N. Andersson, \& D. I. Jones, ApJ 618 (2005) L37

[23] A. L. Watts, N. Andersson, H. Beyer, \& B. F. Schutz, MNRAS 342 (2003) 1156

[24] A. L. Watts, N. Andersson, \& R. L. Williams, MNRAS 350 (2004) 927

[25] M. Saijo \& S. Yoshida, MNRAS 368 (2006) 1429 